\title{
A Review on Segmentation and Feature Extraction by using LBP and PCA for Brain Tumor Detection in MRI
}

\author{
Navjot Kaur \\ Department of Computer Science, DAV Institute of Engineering and Technology, Jalandhar, Punjab
}

\begin{abstract}
Tumor is the premier reason of death in financially created nations and the second most essential reason of death in creating nations. Tumor perceived therapeutically as a dangerous neoplasm, is a broad gathering of a blend of infections, all concerning unregulated cell development. In growth, cells separate and breed generally, framing harmful tumors, and attack adjacent parts of the body. The malignancy may likewise extend to more far off parts of the body through the lymphatic framework or circulation system. Brain tumor is one of the premier explanations behind the ascent in mortality among youngsters and grown-ups. A tumor is a mass of tissue that spreads crazy of the typical powers that manage development. Brain tumors happen when one kind of cell changes from its typical attributes and develops and duplicates in an unusual way.
\end{abstract}

Keywords: Tumor, Brain Tumor, Segmentation, Local Binary Pattern, Principal Component Analysis

\section{INTRODUCTION}

Medicinal imaging is the strategy of making visual portrayals of the inside piece of a body for clinical investigation and restorative intercession to uncover inward structures covered up by the skin and bones, and in addition to analyze and treat the sickness.

Each year, more than 19,000 people in the United States, 10,000 people in Canada and 9000 people in India are diagnosed with brain tumor. The overall incidence of all brain tumors is 100,000 people per year. Although as many as $70 \%$ of children diagnosed with brain tumors survive, but they are often left with long-term side effects. Recently most of the research works are based on early detection of brain tumor and its reliability. This thesis is based on MRI brain tumor detection by segmentation using various soft computing techniques.

The brain is soft spongy mass of tissue. It is protected by the bones of the skull and three thin membranes which are called meninges. A watery fluid called cerebro spinal fluid cushions the brain. This fluid flows through these spaces between the meninges and through spaces within the brain called ventricles. The mass of cells that results from this uncontrolled growth is called a tumor. While some of these tumors do not spread beyond their point of origin, others are capable of spreading large distances, often to other organs and tissues. This process is called metastasis and tumors of that metastasize are called cancers. Brain tumors can either originate from within the brain (primary brain tumors) or from cancer cells that have metastasized from other organs or tissues (secondary brain tumors). Primary brain tumors can be derived from over twenty different cell types that make up the brain. They are named and categorized by the type of cells that produce them. The most common forms of cancer that produce secondary brain tumors include lung, breast, kidney, and skin (melanoma) cancer. While primary brain tumors rarely metastasize outside of the central nervous system (CNS), they all have the propensity to spread within the normal brain, and are all, therefore, cancerous. However, primary brain tumors do vary significantly in terms of how rapidly they grow and spread. Thus, most primary brain tumors are described in terms of grade. Low-grade tumors tend to grow slowly and frequently remain dormant for long periods of time, while high-grade tumors grow and spread rapidly.

If a person has symptoms such as headaches, seizures, vomiting, vision or hearing problems, problems with weakness of the arms, legs or face muscles, and strange sensations in your head or hands that suggest a brain tumor, the doctor may perform one or more of the following procedures: Physical exam ,Neurological exam, CT scan, and MRI scan. MRI provides information that differs from the other imaging techniques. Its major advantage is that it can characterize and discriminate among the tissues using their biochemical (water, iron, fat, and extra vascular blood and its breakdown products) and physical properties. Blood flow, cerebrospinal fluid flow, and contraction and relaxation of organs, both physiologic and pathologic, can be evaluated. Excellent delineation of anatomic structures results from inherent high levels of contrast resolution. In this proposed work MRI brain tumor is detected from unsupervised segmentation technique and various soft computing methods.

Brain tumor plays main role in medical imaging. Brain tumor is a collection of abnormal cells that grows inside of the brain or around the brain in human. Brain tumors are often challenging for doctors to diagnose and then give treatment. 
Diagnosing a brain tumor usually involves several steps. First, Doctor will perform a neurological examination, which among other things includes checking human vision, hearing, balance, coordination and reflexes. Depending on the results of the examination, doctor may suggest one of the following tests: Computerized tomography (CT) scan, Magnetic resonance imaging (MRI) scan, Angiogram, X-rays, and single-photon emission computerized tomography (SPECT). MRI provides much greater contrast between the different soft tissues of the body than computed tomography does making it especially useful in neurological, musculoskeletal, cardiovascular, and oncological imaging.

\section{TYPES OF BRAIN TUMOR}

i. Primary brain tumor: A Primary malignant brain tumor is one that originates in the brain itself. Although primary brain tumors often shed cancerous cells to other sites in the central nervous system (the brain or spine), they rarely spread to other parts of the body. Primary brain tumors are named due to the cell types, from which they are originated.

ii. Secondary brain tumor: A secondary (metastatic) brain tumor occurs when cancer cells spread to the brain from a primary cancer in another part of the body. Secondary tumors are about three times more common than primary tumors of the brain. Secondary or metastatic brain tumors take their origin from tumor cells which spread to the brain from another location in the body.

iii. Benign tumor: A tumor (solid neoplasm) that has self-limiting growth does not conquer other tissues or metastasis. A benign tumor does not contain cancer cells and usually, once removed, does not recur. Most benign brain tumors have clear borders, meaning they do not invade surrounding tissues. These tumors can, however, cause symptoms similar to cancerous tumors because of their size and location in the brain.

iv. Malignant tumor: Malignant brain tumors contain cancer cells. Malignant brain tumors are usually fast growing and conquer surrounding tissues. It very hardly spread to other areas of the body, but may recur after treatment.

Sometimes, brain tumors that are not cancer are called malignant because of their size and location, and the damage they can do with the vital functions of the brain.

\section{BRAIN TUMOR SEGMENTATION}

The critical objective of cerebrum tumor imaging investigation is to separate the patient-particular huge clinical data, and the demonstrative highlights. This data implanted inside the multidimensional picture information, can prompt and oversee intercessions after the infection has been detected and limited, in the long run prompting learning for clinical determination, organizing, and treatment of malady. These procedures can be spoken to diagrammatically as a pyramid [1]. At each level of the pyramid, exact methods are fundamental to process the information, concentrate, mark, and speak to the data.

The primary goal of picture division is to segment a picture into fundamentally unrelated areas to such an extent that every locale is spatially bordering and the pixels inside the district are homogeneous as for a predefined rule [2]. This characterizes the significant constraint of the greater part of the division techniques, particularly when characterizing and depicting "unusual tissue writes", on the grounds that the tumors to be sectioned are anatomical structures which are regularly non-unbending and complex fit as a fiddle, differ incredibly in size and position, and show extensive inconstancy from patient to tolerant.

Brain tumor division strategies can be characterized into three classifications as per the level of the required human communications in particular manual division, self-loader division, and completely programmed division.

i. Manual Segmentation: Manual segmentation of brain tumors includes manual illustration of the limits of the tumor and structures of intrigue, or painting the district of anatomic structures with various marks. Here, human specialists (radiologists/anatomists/prepared technologists) not just make utilization of the data exhibited in the picture yet additionally make utilization of extra information, for example, life systems. Manual depiction requires programming devices with refined graphical UIs to encourage drawing locales of intrigue and picture show. Practically speaking, the choice of the tumor area, which is the Region of Interest (ROI), is a monotonous and tedious undertaking. X-ray scanners create different two-dimensional cross-areas (cuts), and the human master needs to experience the dataset cut by cut for picking the most illustrative ones from which the applicable districts are deliberately depicted. Manual division of cerebrum tumors is likewise ordinarily done based on a solitary picture with power upgrade gave by an infused differentiate operator.

ii. Semiautomatic Segmentation: The mediation of a human administrator is regularly required in self-loader cerebrum tumor division to introduce the strategy, to check the exactness of the outcome, or even to physically rectify the division result. The vast majority of the ebb and flow inquire about is focused at self-loader division of cerebrum tumors with the expectation of having the slightest human cooperation conceivable. The principle segments of an intelligent cerebrum tumor division technique are the computational part, the intuitive part, and the UI. The 
Vol. 8, Issue 5, May 2019

computational part compares to at least one bits of projects equipped for producing a depiction of the tumor which gives a few parameters. The intelligent part is in charge of interceding data between the client and the computational part. It deciphers the result created by the computational part into visual criticism to the client and the information contribution by the client into parameters for the program. The genuine correspondence between the PC and the client is done by means of the yield and information gadgets controlled by the UI. The client breaks down the visual data showed on the screen and responds appropriately, giving criticism to the calculation.

iii. Fully automatic Segmentation: In fully automatic methods, the computer decides the division of tumor with no human collaboration. Completely programmed strategies by and large fuse human insight and earlier learning in the calculations, and are normally created making utilization of delicate registering and model-based methods, for example, deformable models.

\section{FEATURE EXTRACTION TECHNIQUES}

Local Binary Pattern (LBP): It is a feature extracting technique which labels the pixels of an image by thresholding the neighborhood of each pixel and it considers the result as a binary number. Due to its discriminative power and computational simplicity, it becomes a popular approach in various applications. It can be seen as a unifying approach to the traditionally divergent statistical and structural models of texture analysis. Perhaps the most important property of the LBP operator in real-world applications is its robustness to monotonic gray-scale changes caused, for example, by illumination variations. Another important property is its computational simplicity, which makes it possible to analyze images in challenging real-time settings.

LBP is a descriptor for describing the texture of a rectangular block. The LBP operator assigns a value to every pixel of an image in a $3 \times 3$ neighbourhood of a center pixel by comparing it with the center pixel value. If the center pixel is greater than the neighbourhood pixel, it assigns " 1 ", otherwise " 0 ".

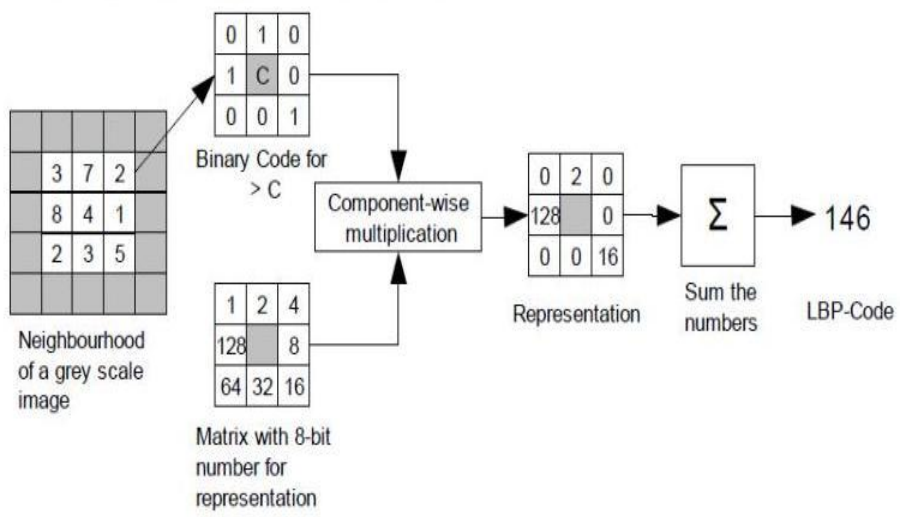

Fig.1: Example of how LBP works

Principal Component Analysis (PCA): It is one of the most successful techniques that have been used in image recognition and compression. Principal Component Analysis is the general name for a technique which uses sophisticated underlying mathematical principles to transforms a number of possibly correlated variables into a smaller number of variables called principal components. PCA is a statistical method under the broad title of factor analysis. The purpose of PCA is to reduce the large dimensionality of the data space (observed variables) to the smaller intrinsic dimensionality of feature space (independent variables), which are needed to describe the data economically. This is the case when there is a strong correlation between observed variables. The jobs which PCA can do are prediction, redundancy removal, feature extraction, data compression, etc. Because PCA is a classical technique which can do something in the linear domain, applications having linear models are suitable, such as signal processing, image processing, system and control theory, communications, etc. Face recognition has many applicable areas. Moreover, it can be categorized into face identification, face classification.

\section{Steps Used in PCA:}

Step 1: Get some data

Step 2: Subtract the mean

Step 3: Calculate the covariance matrix.

Step 4: Calculate the eigenvector and eigenvalues of the covariance matrix.

Step 5: Choosing components and forming a feature vector.

Step 6: Deriving the new dataset. 


\section{BACKGROUND}

Purnima et al. [3] proposed another approach towards this field i.e. Wise Water Droplets calculation. Mind tumor limit discovery is of most extreme significance these days in medicinal line as a great many cases are being enrolled day by day of this deadly tumor. For this reason various division systems are additionally being utilized, for example, Multifractal Texture Estimation, Artificial Bee Colony calculation, Integrated Bayesian Model and so on. Be that as it may, aftereffects of these calculations are very little effective. IWD is a swarm based approach that has been utilized as various critical thinking strategies and because of its inclination to deliver much ideal and effective outcomes, I have instilled it in recognizing the limit of mind tumor in MRI pictures.

Zhang et al. [4] proposed a novel self-loader division calculation to section mind tumors from attractive reverberation imaging (MRI) pictures. Initial, an edge mindful channel is utilized to get the smoothed variant of the first picture. Also, Otsu construct multilevel thresholding is performed in light of the smoothed picture and the first picture, individually. At that point the two division maps are combined by the administer of K Nearest Neighbors (KNN) to acquire the refined division result. The blend of the three stages can be signified as multi-scale Otsu based division. At last, a bi-directional district developing strategy is utilized to portion the mind tumor area around seeds which are embedded by the client. The proposed calculation is tried on MRI-T2 pictures and it produces promising outcome: the fragmented tumor areas are more precise contrasted with those acquired by other cutting edge strategies.

Sehgal et al. [5] acquainted a completely programmed technique with recognize cerebrum tumors. A cerebrum tumor or intracranial neoplasm is shaped when irregular cells get amassed inside the mind. These cells duplicate in an uncontrolled way and harm the mind tissues. Attractive Resonance Imaging (MRI) filters are generally used to analyze cerebrum tumors. Notwithstanding, dividing and identifying the mind tumor physically is a monotonous assignment for the radiologists. Henceforth, there is a requirement for programmed frameworks which yield precise outcomes. The proposed strategy comprises of five phases, viz., Image Acquisition, Preprocessing, Segmentation utilizing Fuzzy C Means procedure, Tumor Extraction and Evaluation. Tumor extraction is completed by utilizing Area and Circularity as a criteria. The outcomes are at long last checked by contrasting them and the physically sectioned Ground Truth. Dice coefficient is likewise figured and the normal dice coefficient esteem got was 0.729 .

Lu et al. [6] proposed anisotropic dispersion and Markov irregular field (MRF) smooth limitation. Mind tumor division strategy is a critical clinical prerequisite for the cerebrum tumor finding and the radiotherapy arranging. Be that as it may, the quantity of bunches is exceptionally hard to characterize for high decent variety in the presence of tumor tissue among the diverse patients and the vague limits about the sores. In our investigation, the nonparametric blend of Dirichlet process (MDP) show is utilized to section the tumor pictures consequently, which can be performed without introduction of the bunching number. Division comes about for the multimodal MR glioma picture groupings demonstrated the properties, for example, precision and processing speed about our calculation exhibits extremely great.

Goel et al. [7] presents a Multispectral approach for division of tumorcenter utilizing three MRI symbolism viz. T1differentiate, T2 and Flair. In all nations the quantity of individuals determined to have mind tumor is expanding quickly. This puts individuals' life in genuine peril. A lot of MRI filters demonstrating cerebrum tumor are as of now being created in centers. The manual procedure for tumor division by Radiologists is very tedious and inclined to human mistakes and inclinations. Subsequently programmed and dependable frameworks to do the errand are required. The proposed calculation for division of cerebrum tumor is free of info parameters and appraisals. So it is totally selfruling. The calculation works in five stages: Image Acquisition, Image Enhancement took after by Segmentation (Fuzzy C Means calculation) at that point Extraction of tumorcenter utilizing Area and Circularity as criteria lastly assessing the outcome with Ground Truth. The mean estimation of dice coefficient for portioning tumorcenter is 0.88 . Subsequently division of tumorcenter utilizing proposed strategy yields proficient outcomes.

Dawngliana et al. [8] proposed hybridized multilevel thresholding and level set technique for programmed division of mind tumor. Division of tumor from attractive reverberation picture (MRI) mind pictures is a developing examination region in the field of therapeutic picture division. As division of mind tumor assumes a vital part for fundamental treatment and arranging of tumor medical procedure. Notwithstanding, division of the cerebrum tumor is as yet an extraordinary test in centers, exceptionally programmed division. The advancement for this paper is to interface the underlying division from multilevel thresholding and concentrate a fine representation utilizing level set technique with morphological activities. The outcomes are contrasted and the current strategy and furthermore with radiologist manual division which affirm the adequacy of this hybridized worldview for mind tumor division.

Akram et al. [9] propose a strategy for programmed mind tumor demonstrative framework from MR pictures. Theoretical - Magnetic reverberation (MR) pictures are an exceptionally helpful instrument to recognize the tumor development in cerebrum yet exact mind picture division is a troublesome and tedious process. The framework comprises of three phases to recognize and fragment a mind tumor. In the primary stage, MR picture of cerebrum is obtained and preprocessing is done to expel the commotion and to hone the picture. In the second stage, worldwide limit division is done on the honed picture to portion the mind tumor. In the third stage, the divided picture is post handled by morphological tasks and tumor veiling so as to evacuate the false portioned pixels. Results and analyses demonstrate that our propose procedure precisely recognizes and sections the mind tumor in MR pictures. 
Edgar et al. [10] introduce a methodical way to deal with describe inconstancy in tumor limits that uses comparability tests as a way to decide if a tumor volume has fundamentally changed after some time. Mind tumor investigation is moving towards volumetric evaluation of attractive reverberation imaging (MRI), giving a more exact portrayal of malady movement to better educate clinical basic leadership and treatment arranging. While a large number of division approaches exist, innate inconstancy in the aftereffects of these calculations may mistakenly show changes in tumor volume. To show these ideas, 32 MRI thinks about from 8 patients were divided utilizing four unique methodologies (factual classifier, locale based, edge-based, information based) to create distinctive areas of enthusiasm speaking to tumor degree. We demonstrated that over all examinations, the normal Dice coefficient for the superset of the diverse strategies was 0.754 (95\% certainty interim $0.701-0.808)$ when contrasted with a reference standard. We show how inconstancy got by various divisions can be utilized to distinguish critical changes in tumor volume between consecutive time focuses. Our examination shows that inconstancy is an intrinsic piece of deciphering tumor division comes about and ought to be considered as a component of the understanding procedure.

Aslam et al. [11] introduced an Improved Edge Detection calculation for mind tumor division. Picture division is utilized to isolate objects from the foundation, and hence it has ended up being an intense apparatus in bio-therapeutic imaging. It depends on Sobel edge identification. It consolidates the Sobel technique with picture subordinate thresholding strategy, and finds distinctive locales utilizing shut shape calculation. At long last tumors are extricated from the picture utilizing force data inside the shut shapes. The calculation is actualized in $\mathrm{C}$ and its execution is estimated equitably and in addition subjectively. Re-enactment comes about demonstrate that the proposed calculation gives better execution over traditional division techniques. For relative investigation, different parameters are utilized to exhibit the predominance of proposed strategy over the traditional ones.

Samriti et al. [12] defer utilizing watershed and differentiation strategy. In therapeutic picture handling mind tumor location is a testing errand. Picture of cerebrum is hard to get it. Commotion and postpone influences the picture precision. In this technique MRI (attractive reverberation imaging) has turned into a valuable therapeutic indicative device for conclusion of cerebrum and other restorative pictures. Picture division is a noteworthy region of medicinal picture handling.

\section{PROPOSED WORK}

An abnormal growth of cells in the brain is called a brain tumor. A brain tumor consists of a collection of abnormally functioning brain cells that have begun to grow and reproduce inappropriately. The uncontrolled growth of group of cells compresses and damages normal brain structures, which causes a variety of neurological symptoms. According to the reports of National Cancer Institute, Primary brain tumors are the leading cause of tumor cancer deaths in children, now surpassing acute lymphoblast leukemia and are the third leading cause of cancer death in young adults ages 20 to 39. There are more than 120 different types of brain tumors, making effective treatment very complicated. As per classification system defined by World Health Organization (WHO), brain tumor is named for the cell type of origin. Brain tumors can either originate from within the brain or from cancer cells that have metastasized from other organs or tissues. Various techniques are developed in the past to detect brain tumor. Proposed work is basically to design a Hybrid Fuzzy Genetic Classification algorithm for Brain Tumor. For segmentation we are using K-means Clustering and Otsu Method.From MRI using LBP and PCA for Feature Extraction and Fuzzy Genetic for Classification is proposed. Various parameters like computation time, Mean, Standard deviation, Entropy, RMS and Accuracy are used to evaluate the results.

Techniques mentioned in literature survey don't have scale invariant features hybridizing with Eigen values and Eigen vector for better performance of system. Individually $\mathrm{K}$-means clustering is good for segmentation but not for optimized feature extraction it's only when k-means used along with LBP and PCA. Previous techniques use matching algorithms but we add Fuzzy Genetic to train and test large number of dataset. Using existing methods we can't achieve efficient results that are why we proposed a hybridized system which overcome all the problems and performance issues. Proposed technique is to design a Hybrid Fuzzy Genetic classification algorithm for Tumor detection. Various parameters would evaluate the results of the proposed work and it would detect brain tumor more efficiently and effectively by using K-means clustering and Otsu method.

\section{CONCLUSION}

The current technological development in Digital image processing and analysis has found many applications in various fields like satellite image analysis, medical image analysis and other industrial applications related to process automation. Recently, researchers have developed automated computer based analysis systems for tissue classification and analysis, but none of them provide precise morphological information for the cancerous tumors. 


\section{International Journal of Advanced Research in Computer and Communication Engineering}

Vol. 8, Issue 5, May 2019

\section{REFERENCES}

[1]. Wong, K 2005, 'Medical image segmentation: methods and applications in functional imaging', Handbook of Biomedical Image Analysis, vol.2, pp.111-182.

[2]. Bhandarkar, S, Koh, J \& Suk, M1997, 'Multiscale image segmentation using a hierarchical self-organizing map', Neurocomputing, vol.14, pp.241-272.

[3]. Purnima, Vikas Wasson, "BRAIN TUMOR BOUNDARY DETECTION USING IMPROVED OPTIMIZATION TECHNIQUE", International Journal of Computer Engineering and Applications, Volume VI, Issue III, June 14, pp. 69-75.

[4]. Xiaoli Zhang, Xiongfei Li, Hongpeng Li, and YuncongFeng, "A SEMI-AUTOMATIC BRAIN TUMOR SEGMENTATION ALGORITHM", IEEE.

[5]. AasthaSehgal, ShashwatGoel, ParthasarathiMangipudi, AnuMehra, DevyaniTyagi, "Automatic Brain Tumor Segmentation And Extraction In MR Images", IEEE, 2016, pp. 104-107.

[6]. Yisu Lu, Wufan Chen, "Automatic Multimodal Brain-tumor Segmentation", IEEE, International Conference on Instrumentation and Measurement, Computer, Communication and Control, 2015, pp. 939-942.

[7]. ShashwatGoel, AasthaSehgal, ParthasarathiMangipudi, AnuMehra, "Brain Tumor Segmentation in Multispectral MR Images", IEEE, International Conference on Signal Processing and Integrated Networks, 2017, pp. 1-4.

[8]. MalsawmDawngliana, Daizy Deb, MousumHandique, Sudipta Roy, "Automatic Brain Tumor Segmentation in MRI: Hybridized Multilevel Thresholding and Level Set”, IEEE, International Symposium on Advanced Computing and Communication, 2015.

[9]. M. UsmanAkram, AnamUsman, "Computer Aided System for Brain Tumor Detection and Segmentation”, IEEE, 2011.

[10]. Edgar A. Rios Piedra, Ricky K. Taira, Suzie El-Saden, "Assessing Variability in Brain Tumor Segmentation to Improve Volumetric Accuracy and Characterization of Change", IEEE, 2016, pp. 380-383.

[11]. AsraAslam, Ekram Khan, M.M. Sufyan Beg, "Improved Edge Detection Algorithm for Brain Tumor Segmentation", International Symposium on Computer Vision and the Internet, 2015.

[12]. Samriti, Mr.Paramveer Singh, "Brain Tumor Detection Using Image Segmentation", International Journal of Engineering Development and Research, 2016

[13]. Swati Dubey, LakhwinderKaur, “An Assessment on Automatic Brain Tumor Detection”, International Journal of Computer Science and Mobile Computing, Vol.5 Issue.3, March- 2016, pp. 236-243.

[14]. Arati Kothari, B. Indira, "An Overview on Automated Brain Tumor Segmentation Techniques", International Journal of Computer Trends and Technology, 2016.

[15]. NileshBhaskarraoBahadure, Arun Kumar Ray, and Har Pal Thethi, "Image Analysis for MRI Based Brain Tumor Detection and Feature Extraction Using Biologically Inspired BWT and SVM", International Journal of Biomedical Imaging, 2017.

[16]. M.Jasmine, "A Relative Study of MRI Based Brain Tumor Segmentation Methods", International Journal of Pure and Applied Mathematics, 2017 